\title{
Simple distributed fiber sensor based on Brillouin gain spectrum analysis
}

\author{
Marc Niklès, Luc Thévenaz, and Philippe A. Robert \\ Metrology Laboratory, Swiss Federal Institute of Technology, CH-1015 Lausanne, Switzerland
}

Received November 16, 1995

\begin{abstract}
A novel configuration of a distributed fiber sensor by Brillouin gain analysis has been developed for temperature and strain monitoring. It uses a single laser source, and the required light signals are all generated with an electro-optic modulator, resulting in high stability and excellent reliability of the measuring setup. Measurement of the induced strain in a wound fiber is presented as a demonstration of the system performance. (c) 1996 Optical Society of America
\end{abstract}

Brillouin gain spectrum measurement along an optical fiber has recently been the subject of much interest because of its potential use for strain monitoring in installed cables ${ }^{1}$ and distributed temperature sensing. ${ }^{2}$ This measurement requires two light waves propagating in opposite directions through the fiber, because stimulated Brillouin scattering (SBS) amplification is possible only in the backward direction for phase-matching reasons. One light wave pumps the medium, and the other acts as a probe signal, experiencing amplification when its frequency lies within the Brillouin gain spectral range. The Brillouin gain spectrum (BGS) is downshifted with respect to the pump light frequency by the Brillouin frequency shift $\nu_{B}$, which ranges from 11.5 to $13 \mathrm{GHz}$ at a pump wavelength near $1300 \mathrm{~nm}$, depending on the fiber's refractive-index profile. ${ }^{3}$ This frequency shift is given by $\nu_{B}=2 n V_{a} / \lambda_{0}$, where $V_{g}$ is the acoustic velocity within the fiber, $n$ is the refractive index, and $\lambda_{0}$ is the vacuum wavelength of the incident light wave. The sensitivity of $\nu_{B}$ to temperature and strain applied to the fiber makes SBS highly suitable for sensing applications. Measuring the BGS at different locations along the sensing fiber gives access to $\nu_{B}$ and consequently to the local strain or temperature. Distributed measurements can be achieved with a modified optical time-domain reflectometry technique. Most of the methods reported so far either use two distinct laser sources, ${ }^{1,2}$ causing problems when the fiber ends are remote, or employ a single laser source at the expense of setup complexity. ${ }^{4}$

A novel configuration is presented here, based on the pump-and-probe technique, that has the following advantages: (1) few optical elements are required, (2) a single laser source is used, (3) a single fiber end is accessed, and (4) only pulsed signals propagate throughout the fiber.

A schematic diagram of the experimental setup is shown in Fig. 1. The operation of this setup relies on two features. ${ }^{5}$ First, the pump and the probe are pulsed signals that both propagate back and forth through the sensing fiber, using a reflection at the far end. The pump pulse provides gain to the probe signal during its forward propagation through the SBS process, and the probe pulse is amplified on the way back. Reflection of only a few percent is necessary at the fiber far end, so Fresnel reflection is actually sufficient. The amplification actually occurs only at the crossing point of the pulses, preventing pump power from being depleted before the interaction takes place. In addition, depletion owing to noise amplification is negligible; the effective net Brillouin gain ranges from 1 to 5, resulting in a negligible amplified noise level compared with those for pump and probe intensities in the milliwatt and microwatt ranges, respectively. The second and more important feature is the use of an ultrawideband integrated $\mathrm{LiNbO}_{3}$ intensity electro-optic modulator (EOM). This modulator is the key element of the setup, as it is used on the one hand for pulsing the cw laser light from a $150-\mathrm{mW}$ Nd:YAG laser to form the pump signal and on the other hand for the generation and frequency tuning of the probe signal. The frequency shift of the laser light is achieved simply by application of a microwave signal on the EOM electrodes. This creates sidebands in the laser spectrum, as shown in Fig. 2. When the modulation frequency $f_{m}$ is close to the Brillouin frequency shift $\nu_{B}$, the first lower sideband lies in the BGS generated by the pump and is amplified through the SBS process. ${ }^{6}$ As a matter of fact, one can determine the BGS by simply sweeping the modulation frequency $f_{m^{\prime}}$ and recording the probe intensity. The frequency spacing between the first upper and the first lower sidebands is twice the frequency modulation $f_{m^{\prime}}$ (approximately $24 \mathrm{GHz}$ ) and corresponds to a wavelength separation of $0.15 \mathrm{~nm}$ at $1.32 \mu \mathrm{m}$. Inasmuch as the first upper sideband is not relevant for the measurement and even has a nega-

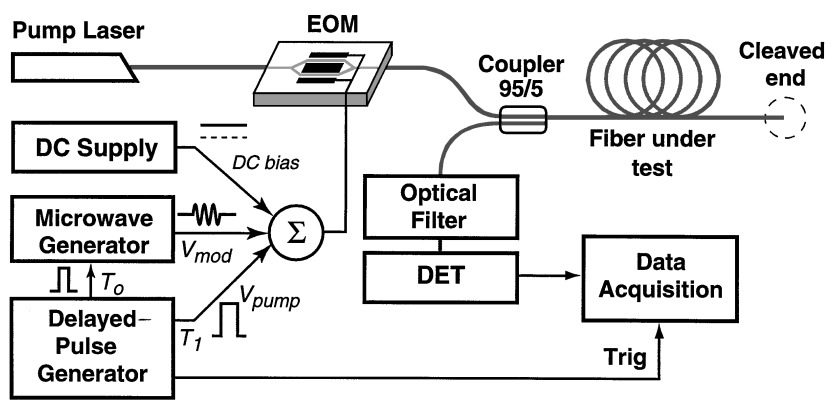

Fig. 1. Experimental setup for distributed BGS measurements, which uses an EOM to generate the interacting optical signals. DET, detector. 


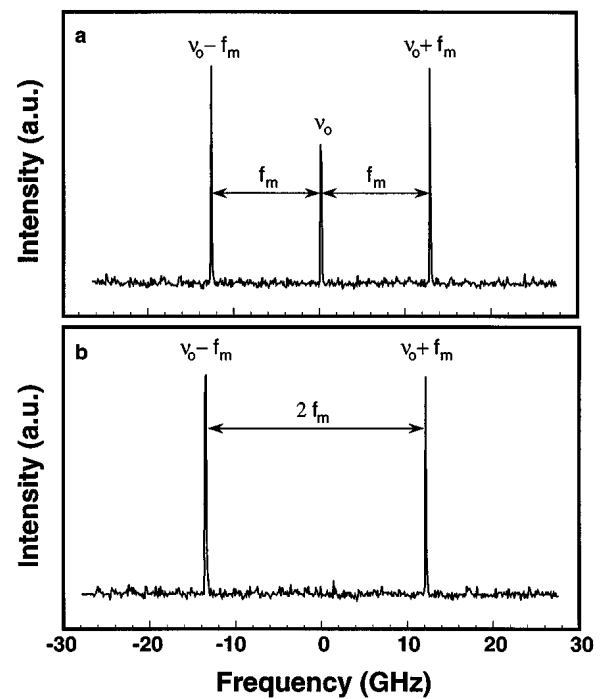

Fig. 2. Fabry-Perot spectra of a single-frequency laser modulated by a, an electro-optic carrier and first-order sidebands and b, suppression of the carrier by setting the dc bias of the modulator electrodes at the zero-transmission point.

tive effect on the contrast, it can be suppressed with a Fabry-Perot or any narrow-band filter. This technique makes the control of the probe optical frequency convenient and reliable by adjusting the microwave modulation signal frequency. In addition one can easily pulse the probe by gating the microwave generator output. The dc bias setting on the EOM just determines the amount of transmitted amplitude of the fundamental frequency, as can be seen from Fig. 2.

Figure 3 shows the transmitted intensity of the EOM as a function of the modulation voltage and the timing of the electrical driving signals applied to the EOM electrodes. First the dc bias is set so no light is transmitted through the EOM. At time $T_{0}$ and for a short time $\tau_{s}$ the EOM modulates the laser light at frequency $f_{m^{\prime}}$ to generate a probe pulse with an optical spectrum identical to that of Fig. $2 \mathrm{~b}$. At time $T_{1}$ we switch on the modulator on by applying an electrical pulse to the EOM electrodes, creating a pump pulse at the laser frequency $\nu_{0}$. A digital delay generator is used to adjust the delay $\Delta T=T_{1}-T_{0}$ between the probe and the pump pulses so they cross at a definite location, and every position along the sensing fiber can be addressed in this way.

Because this technique uses a single laser and a modulator for the generation of the pump and the probe signals, it ensures an inherent stability of their frequency difference. Measurements of the distribution of the Brillouin frequency shift along a sensing fiber are performed with excellent resolution, and the measured standard deviation of the Brillouin shift frequency $\nu_{B}$ is $300 \mathrm{kHz}$. The spatial resolution is equivalent to the length of the fiber segment where the interaction actually takes place, that is, the overlapping distance of the pump and probe optical pulses. Inasmuch as the Brillouin frequency shift $\nu_{B}$ is much smaller than the optical frequencies, pump and probe pulses are reasonably assumed to have the same group velocity $v_{g}$. Thus the spatial resolution $L_{\text {int }}$ is simply given by $L_{\text {int }}=1 / 2 v_{g}\left(\tau_{P}+\tau_{S}\right)$, where $\tau_{P}$ and $\tau_{S}$ are the pump and the probe pulse widths, respectively. For a given pump power $P_{P}$ the ultimate spatial resolution is determined by the narrowest pump pulse width that leaves a sufficient gain for a BGS measurement to be performed with minimum contrast. Actually, the probe grows exponentially as long as it overlaps the pump pulse, assuming negligible pump depletion. Under this assumption the overall gain $G_{B}$ experienced by the probe signal shows no dependence on the probe amplitude and duration. The relevant amplification length corresponds to the probe's traveling length $L_{g}$ where the pump pulse is present, i.e., $L_{g}=(1 / 2) v_{g} \tau_{p}$. Hence

$$
G_{B}=\exp \left(\frac{g_{B} P_{P} \tau_{P} v_{g}}{2 A_{\text {eff }}}\right)
$$

where $g_{B} \approx 2 \times 10^{-11} \mathrm{~m} / \mathrm{W}$ is the effective Brillouin gain coefficient in fibers ${ }^{3}$ and $A_{\text {eff }}$ the effective core area. A minimum contrast corresponds to a minimum $G_{B}$ that depends only on the product $P_{P} \tau_{P}$ for a given fiber, so the pump power determines the minimum possible pulse width $\tau_{P}$ and thus the spatial resolution. The effective gain is decreased in case of pump and probe polarization mismatch, but the interaction length in the tens-of-meters range is usually much larger than the beat length in the meter range. Deventer and Boot ${ }^{7}$ demonstrated that $g_{B}$ ranges from $1 / 3$ to $2 / 3$ of its maximum value in this case, leaving a sufficient gain for measurements without tuning the polarization. However, in future systems with a high spatial resolution in the meter range the measurement contrast is likely to fade locally and may require an additional polarization control. Because the issue is to maximize the pump power in the fiber under test, an unbalanced coupler $(95 / 5)$ is used. The standard insertion loss of the $\mathrm{LiNbO}_{3}$ modulator $(4.7 \mathrm{~dB})$ brings a power penalty, limiting the available pump power to $45 \mathrm{~mW}$ in the fiber. To optimize the spatial resolution (i.e., minimize $L_{\text {int }}$ ) we reduced the probe width to $50 \mathrm{~ns}$. For a standard optical fiber the best

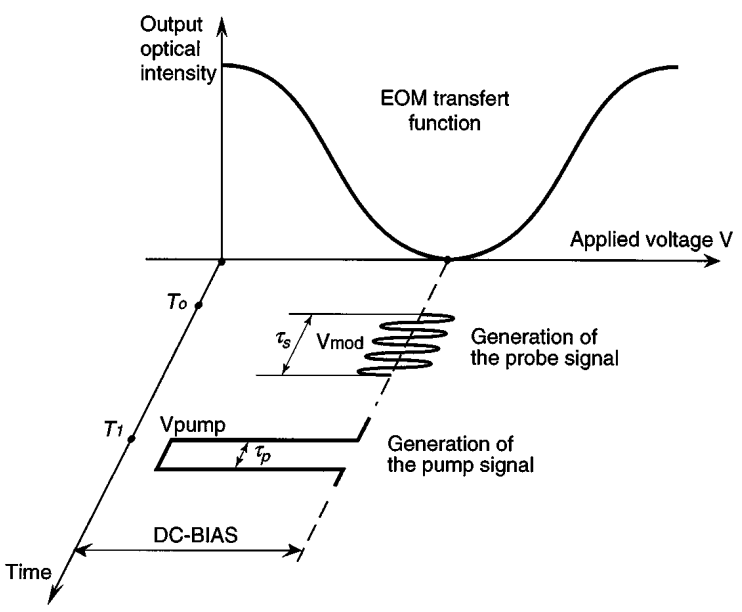

Fig. 3. Schematic diagram of the EOM transmitted optical intensity as a function of the applied voltage together with the sequence of the electrical driving signals as a function of time. Modulation leaves a probe signal containing frequencies at $\nu_{0}+f_{m}$ and $\nu_{0}-f_{m}$. 


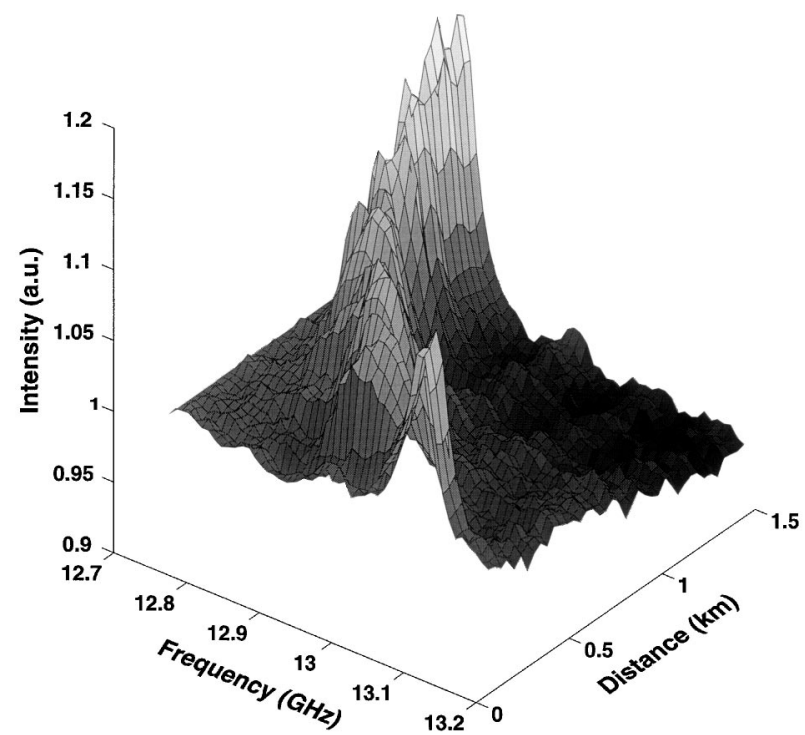

Fig. 4. Distributed Brillouin gain profile of a 1.4-km fiber wound on a 16-cm-diameter drum with high winding tension.

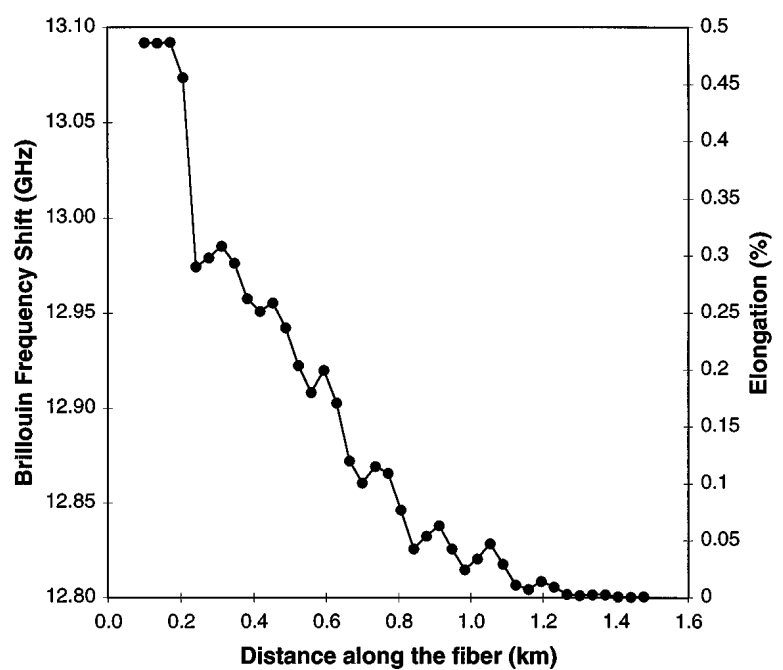

Fig. 5. Local Brillouin frequency shift and corresponding tensile strain variations along the same $1.4-\mathrm{km}$ fiber. Each period of the waved distribution corresponds to one 140-m layer of fiber wound on the drum.

spatial resolution obtained so far is $45 \mathrm{~m}$, corresponding to 400-ns pump pulse width. The net gain $G_{B}$ is greater for fibers with smaller core diameters, such as dispersion-shifted fibers, for which the spatial resolution is improved to $35 \mathrm{~m}$.

This configuration has been successfully tested for distributed temperature ${ }^{6}$ and strain sensing, and results are shown here for local strain measurements. The strain dependence of the Brillouin frequency $\nu_{B}$ was perfectly linear over a $1 \%$ range of elongation, re- sulting in a frequency shift of $600 \mathrm{MHz}$ per percent of elongation. ${ }^{8}$ Figure 4 shows the effect of tensile strain on a $1.4-\mathrm{km}$ standard single-mode fiber wound on a 16-cm-diameter drum by a high winding tension. The BGS was measured with a 50-m spatial resolution over the whole fiber length every $40 \mathrm{~m}$ starting from the center of the drum. Figure 5 represents the variation of the Brillouin frequency shift $\nu_{B}$ along the fiber length. The first layer of fiber experiences a tensile strain as high as $0.5 \%$. Then the strain gradually decreases, reaching zero tension after $\sim 1.2 \mathrm{~km}$, owing to fiber sliding and the resultant released strain. Each fiber layer on the drum (approximate length $140 \mathrm{~m}$ ) experiences a decreasing strain and can be clearly distinguished by the steps shown in Fig. 5 .

In conclusion, a new configuration for distributed strain and temperature measurement based on Brillouin gain spectrum analysis has been developed. It presents the main advantage of requiring few optical elements and only one laser source and consequently shows great inherent stability. It is therefore promising for the further development of an instrument, especially when one considers that access to only one fiber is needed. The best spatial resolution obtained so far is $45 \mathrm{~m}$ for standard optical fibers and $35 \mathrm{~m}$ for dispersion-shifted fibers. Increasing the pump power will result in a proportionally improved spatial resolution. The measurement resolution is approximately $5 \times 10^{-6}$ for strain and $0.25^{\circ} \mathrm{C}$ for temperature, thanks to the high stability of the system.

The authors are grateful to the Swiss Telecom, Cortaillod Cossonay Câbles S.A., and Swiss Electrical Energy Research Funding (PSEL) for their financial support and valuable discussions.

\section{References}

1. T. Horiguchi, T. Kurashima, and M. Tateda, IEEE Photon. Technol. Lett. 2, 352 (1990).

2. X. Bao, D. J. Webb, and D. A. Jackson, Opt. Lett. 18, 552 (1993).

3. L. Thévenaz, M. Niklès, and P. Robert, in Symposium on Optical Fiber Measurement, Natl. Inst. Stand. Technol. Spec. Publ. 864, 211 (1994).

4. K. Shimizu, T. Horiguchi, Y. Koyamada, and T. Kurashima, Opt. Lett. 18, 185 (1993).

5. M. Niklès, L. Thévenaz, and P. Robert, in Optical Fiber Communication Conference, Vol. 4 of 1994 OSA Technial Digest Series (Optical Society of America, Washington, D.C., 1994), paper WF1.

6. M. Niklès, L. Thévenaz, and P. Robert, in Optical Fiber Sensor Conference, Proc. SPIE 2360, 138 (1994).

7. M. O. van Deventer and A. J. Boot, J. Lightwave Technol. 4, 585 (1994).

8. M. Niklès, C. Gabioud, L. Thévenaz, and P. Roberts, presented at the 3rd Optical Fibre Measurements Conference, Liège, Belgium, September 25-26, 1995. 